\title{
No Incidence of BRAF Mutations in Salivary Gland Carcinomas-Implications for Anti-EGFR Therapies
}

\author{
Regine Dahse, ${ }^{1}$ Katrin Kromeyer-Hauschild, ${ }^{2}$ Alexander Berndt, ${ }^{3}$ and Hartwig Kosmehl ${ }^{1}$ \\ ${ }^{1}$ Institute of Pathology, HELIOS Klinikum Erfurt, Nordhauser Str. 74, 99089 Erfurt, Germany \\ ${ }^{2}$ Institute of Human Genetics and Anthropology, Friedrich Schiller University of Jena, Kollegiengasse 10, 07740 Jena, Germany \\ ${ }^{3}$ Institute of Pathology, Friedrich Schiller University of Jena, Ziegelmühlenweg, 07740 Jena, Germany
}

Correspondence should be addressed to Regine Dahse, rdahse@erfurt.helios-kliniken.de

Received 7 April 2009; Accepted 7 May 2009

Recommended by Fernando Schmitt

\begin{abstract}
BRAF is the main effector of KRAS in the RAS-RAF-MAPK axis, a signaling pathway downstream of EGFR. The activation of this cascade is an important pathway in cancer development and is considered a key pathway for therapeutic molecules. Recent studies in metastatic colorectal cancer found that an oncogenic activation of BRAF by a point mutation in exon 15 (V600E) could bypass the EGFR-initiated signaling cascade with the effect that patients bearing the mutant BRAF allele are not likely to benefit from EGFR-targeted therapies. We designed an allele-specific PCR and screened 65 salivary gland carcinoma (SGC) of the main histopathological types for the BRAF V600E mutation. All 65 SGC in this cohort (100\%) presented the BRAF wildtype. In a previous study, we found a KRAS wildtype in $98.5 \%$ of SGC. These findings imply that SGC rarely acquires mutations that result in a constitutive activation of the signaling cascade downstream of EGFR and this pleads in favor of further therapeutic trials with EGFR-targeting monoclonal antibodies.
\end{abstract}

Copyright () 2009 Regine Dahse et al. This is an open access article distributed under the Creative Commons Attribution License, which permits unrestricted use, distribution, and reproduction in any medium, provided the original work is properly cited.

\section{Introduction}

Malignant salivary gland neoplasms account for $<0.5 \%$ of all malignancies and approximately $3-5 \%$ of all head and neck cancers [1]. Progress in understanding the cell biology of salivary gland carcinomas (SGCs) and detecting vulnerable molecular pathways may lead to the development of new targeted therapy options in these rare cancers with poor prognosis. The EGFR signaling cascade is considered a possible key pathway for therapeutic molecules. Anti-EGFR agents include (I) monoclonal antibodies (cetuximab or erbitux, panitumumab) which block the binding of natural EGFR ligands like EGF or TGF- $\alpha$ resulting in inhibition of downstream signal-transduction pathways and (II) small molecule tyrosine kinase inhibitors (TKIs) which act by binding the ATP pocket within the kinase domain of the EGFR and impairing its catalytic activity (gefitinib, erlotinib, lapatinib). Downstream signaling pathways triggered by EGFR include the RAS-RAF-extracellular signal-regulated kinase/mitogen activated protein kinase (MEK/MAPK) pathway, which is mainly correlated to cell proliferation, and the P13K-PTENAKT axis.
Recently, we were able to demonstrate that frequent EGFR overexpression and the absence of drug-resistance EGFR mutations in SGC plead in favor of further therapeutic trials with EGFR-targeting monoclonal antibodies. One of the signaling effectors downstream of EGFR, KRAS, was shown by us to be rarely mutated in SGC $[2,3]$. Wildtype KRAS is one of the clinically proven prerequisites for a successful anti-EGFR therapy and therefore anti-EGFR monoclonal antibodies are approved only for metastatic colorectal cancer patients whose tumors display wildtype KRAS.

In the absence of KRAS mutations, resistance to antiEGFR treatments could be caused by alterations of other members of the RAS-RAF-MAPK pathway. BRAF (v-raf murine sarcoma viral oncogene homolog B1), a serine/ threonine kinase, is the downstream effector of KRAS in the RAS-RAF-MAPK signaling pathway. A somatic mutation (V600E) in exon 15 of BRAF has been identified in multiple human cancers with a mutation rate of $66 \%$ in malignant melanomas [4] and at lower frequency in other human carcinomas. Recently, it was demonstrated that wildtype 
BRAF is required for the response of patients with metastatic colorectal cancer to cetuximab and panitumumab [5].

The aim of this study was to determine the BRAF V600E mutation frequency in a large cohort of SGCs of the main histopathological types and to design an allele-specific PCR as an effective screening method.

\section{Materials and Methods}

2.1. Tissue Specimens. Surgically removed, formalin-fixed tumor samples were obtained from 65 patients ( 35 males and 30 females with a median age at diagnosis of 55 years) treated with the histopathological diagnosis of an SGC according to the WHO classification [6]. All patients received surgery and postoperative radiation therapy in selected cases. EGFRtargeted therapy was not applied. The study cohort consisted of adenoid cystic carcinoma $(n=25)$ mucoepidermoid carcinoma $(n=10)$, myoepithelial carcinoma $(n=8)$, acinic cell carcinoma $(n=12)$ and adenocarcinoma ex pleomorphic adenoma $(n=10)$.

2.2. DNA Isolation. Genomic DNA was extracted and pooled from oral mucosa samples of five healthy individuals. This pooled DNA was used as a normal DNA control for the development of the PCR assay. Heterozygous mutant control DNA was extracted from cells of the colorectal cancer cell line HT 29 which contains the heterozygous BRAF V600E mutation. DNA from the tumor specimen was isolated after microdissecting apprpriate tumor areas.

2.3. Design of an Allele-Specific PCR for the BRAF V600E Mutation. The basis for discrimination using allele-specific PCR is that a PCR primer mismatched at its $3^{\prime}$ end with the DNA template will react less efficiently than one that is entirely complementary. Our allele-specific multiplex PCR was designed with one common forward (BF) and two separate reverse primers ( $\mathrm{BR}$ and $\mathrm{BMu})$ :

\section{BF: 5' -CTCTTCATAATGCTTGCTCTGATAGG-3', BR: 5' -AGTTGAGACCTTCAATGACTTTCTAGT-3', BMu: 5'-CCCACTCCATCGAGATTTCT-3'.}

The forward primer $\mathrm{BF}$ and the reverse primer $\mathrm{BR}$ amplify a $273 \mathrm{bp}$ fragment of both mutant and wildtype alleles and thus serve as amplification control. The second reverse primer $(\mathrm{BMu})$ is specific for the mutated allele at the $3^{\prime}$ end. This primer together with BF generates an $143 \mathrm{bp}$ product only in the presence of the V600E (GTG > GAG) mutation (Figure 1(a)).

A series of annealing temperatures $\left(52^{\circ} \mathrm{C}-60^{\circ} \mathrm{C}\right)$, primer concentrations $(0.1-0.4 \mu \mathrm{mol} / \mathrm{L})$, and $\mathrm{Mg}^{2+}$ concentrations $(1.5-3.5 \mathrm{mmol} / \mathrm{L})$ were tested.

2.4. BRAF Mutational Analysis. PCR reactions for screening SGC were run at a final volume of $25 \mu \mathrm{L}$. Reactions consisted of: $80-100 \mathrm{ng}$ genomic DNA; $200 \mu \mathrm{mol} / \mathrm{L} \mathrm{dNTP;} 0.1 \mu \mathrm{mol} / \mathrm{L}$ of primers; $1.5 \mathrm{mmol} / \mathrm{L} \mathrm{MgCl}_{2} ; 0.5 \mathrm{U}$ Taq polymerase (Roche Diagnostics, Penzberg, Germany). Final cycling conditions were as follows: 5 minutes of denaturing at $94^{\circ} \mathrm{C}$ and 30

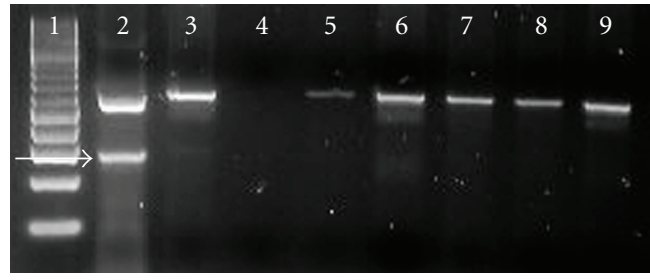

(a)

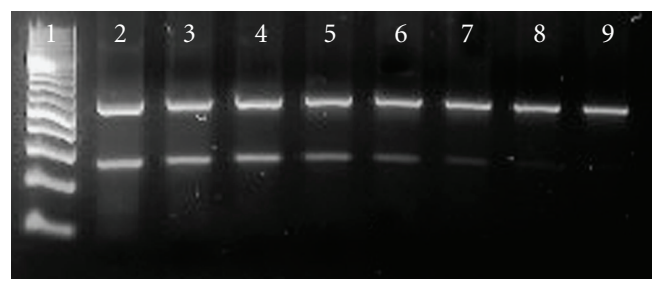

(b)

Figure 1: Allele-specific PCR for the detection of the BRAF V600E mutation (2.5\% agarose gel). (a) The $273 \mathrm{bp}$ fragment of both mutant and wildtype alleles is always amplified and serves as amplification control. The smaller PCR product (143 bp; arrow) is amplified only in the presence of the V600E (GTG>GAG) mutation. Lane 1: Molecular Weight marker; lane 2: heterozygous mutant DNA control (HT 29); lane 3: homozygous wildtype DNA control; lane 4: no template PCR control (water). Lanes 5-9: analysis of tumor samples. The SGC samples in lanes 5-9 have the BRAF V600E wildtype sequence. (b) Serial dilution of HT 29 DNA in normal wildtype DNA. The allele-specific PCR is able to detect the $143 \mathrm{bp}$ mutation specific fragment in an 15-fold excess of normal DNA. Lane 1: Molecular Weight marker; lane 2: heterozygous mutant DNA control [HT 29] undiluted; lanes 3-9: serial dilutions HT 29/WT DNA (starting from 1:2 up to1:64).

cycles of $94^{\circ} \mathrm{C}$ for 30 seconds, annealing $55^{\circ} \mathrm{C}$ for 45 seconds and $72^{\circ} \mathrm{C}$ for 60 seconds. A volume of $10 \mu \mathrm{L}$ of the PCR products was electrophoresed on a standard $2.5 \%$ agarose gel stained with SYBR-Green I for visualization under UV light.

\section{Results}

The allele-specific PCR for the detection of the BRAF V600E mutation demonstrated high specificity (i.e., detection of only the normal or only the mutant allele), high sensitivity (i.e., no spurious PCR fragments), and acceptable yield. All 65 SGC in this cohort (100\%) presented the BRAF wildtype (95\% exact confidence limit 0-0.07). The $273 \mathrm{bp}$ PCR fragment was always amplified confirming the integrity of the isolated DNA from clinical tissue samples (Figure 1(a)).

To test the sensitivity of the mutation-specific PCR, we made a serial dilution of HT 29 DNA (which contains the heterozygous BRAF V600E mutation), in control DNA with wildtype BRAF. Less than $6 \%$ of mutant DNA was reproducibly detected (Figure $1(\mathrm{~b})$ ).

No BRAF V600E mutation was detected in additionally screened DNA samples from microdissected normal tissue adjacent to the tumor cells (5 cases). 


\section{Discussion and Conclusions}

The activation of the EGFR-RAS-RAF signaling cascade is an important pathway in cancer development and is considered a key pathway for therapeutic molecules. EGFR transmits signals to the nuclei instructing cancer cells to proliferate and metastasize, and KRAS and BRAF are those downstream signaling molecules. Anti-EGFR therapies interrupt the cancer-triggering signaling cascade, however, if the KRAS or the BRAF gene is mutated, their proteins are locked into an active conformation, regardless of whether the EGFR is therapeutically blocked.

Cetuximab (erbitux) has already been tested in two phase II studies in patients with recurrent and/or metastatic SGC including mainly ACC, a cancer with generally poor outcome. In $50 \%$ of patients, clinical benefit (i.e., response or stable disease for 6 month) could be achieved [1,7]. Gefitinib was associated with a $53 \%$ stable disease rate (10/19) in ACC, but had no effects on patients with salivary duct tumors and mucoepidermoid cancer [8]. Lapatinib, inhibiting ErbB1 and ErbB2 tyrosine kinases, was studied in a phase II trial and stabilized disease for greater than 6 months in $47 \%$ of ACC patients [9].

Key molecules of the EGFR-RAS-RAF signaling cascade and predictive markers of treatment outcome under antiEGFR therapies have not been comprehensively examined in SGC. Investigations of the mutation status of proteins in the cascade downstream of EGFR identified markers for EGFR-targeted therapy in colorectal cancer. In recent studies, wildtype BRAF as well as wildtype KRAS and intact PTEN PIK3CA were found to be required for the response of colorectal cancer patients treated with cetuximab or panitumumab $[5,10,11]$.

We were able to demonstrate in this and in a former study that KRAS and BRAF mutations seem to be extremely rare in SGC. These findings imply that salivary gland carcinomas which rarely acquire mutations that result in constitutive activation of the signaling cascade downstream of EGFR may be good candidates for anti-EGFR therapies. Molecular analyses of alternative members of the EGFR signaling cascade, such as Akt-1 and MEK-1, may further contribute to elucidating predictive markers of treatment outcome under anti-EGFR therapies in SGC.

Because of its universal availability as a standard methodology in molecular medicine, we designed a BRAF mutation screening assay based on PCR. Allele-specific PCR, also known as Amplification Refractory Mutation System (ARMS), is a well-established method for discriminating between different alleles at specific loci resulting from single base mutations $[12,13]$. We used the methodology to establish an assay with three PCR primers which allows the discrimination of allele-specific PCR fragments by agarose gel electrophoresis without the need of capillary electrophoresis devices. With our assay, the specificity was incorporated into the amplification reaction itself. Interpretation of the results can be made by simple visual inspection of the stained gel to determine whether or not a specific primer pair amplified a fragment with the template DNA. Because microdissected tumor areas were used for the allele-specific
PCR, and a control amplification was incorporated into the PCR reaction to ensure DNA integrity, we can exclude false negative results. So far, genomic screening for BRAF mutations has been based mainly on direct sequencing. Our protocol provides an alternative rapid, sensitive, and costeffective BRAF screening method.

\section{References}

[1] A. Milano, F. Longo, M. Basile, R. V. Iaffaioli, and F. Caponigro, "Recent advances in the treatment of salivary gland cancers: emphasis on molecular targeted therapy," Oral Oncology, vol. 43, no. 8, pp. 729-734, 2007.

[2] R. Dahse, O. Driemel, S. Schwarz, et al., "Epidermal growth factor receptor kinase domain mutations are rare in salivary gland carcinomas," British Journal of Cancer, vol. 100, no. 4, pp. 623-625, 2009.

[3] R. Dahse, O. Driemel, S. Schwarz, K. Kromeyer-Hauschild, A. Berndt, and H. Kosmehl, "KRAS status and epidermal growth factor receptor expression as determinants for antiEGFR therapies in salivary gland carcinomas," Oral Oncology. In press.

[4] H. Davies, G. R. Bignell, C. Cox, et al., "Mutations of the BRAF gene in human cancer," Nature, vol. 417, no. 6892, pp. 949954, 2002.

[5] F. Di Nicolantonio, M. Martini, F. Molinari, et al., "Wildtype BRAF is required for response to panitumumab or cetuximab in metastatic colorectal cancer," Journal of Clinical Oncology, vol. 26, no. 35, pp. 5705-5712, 2008.

[6] L. Barnes, W. Eveson, P. Reichart, and W. Sidransky, "Pathology and genetics of head and neck tumours," in World Health Organization Classification of Tumours, pp. 210-242, IARC Press, Lyon, France, 2005.

[7] L. D. Locati, P. Bossi, F. Perrone, et al., "Cetuximab in recurrent and/or metastatic salivary gland carcinomas: a phase II study," Oral Oncology. In press.

[8] B. S. Glisson, G. Blumenschein, M. Francisco, J. Erasmus, R. Zinner, and M. Kies, "Phase II trial of gefitinib in patients with incurable salivary gland cancer," Journal of Clinical Oncology, vol. 23, no. 16S, p. 5532, 2005.

[9] M. Agulnik, E. W. E. Cohen, R. B. Cohen, et al., "Phase II study of lapatinib in recurrent or metastatic epidermal growth factor receptor and/or erbB2 expressing adenoid cystic carcinoma and non-adenoid cystic carcinoma malignant tumors of the salivary glands," Journal of Clinical Oncology, vol. 25, no. 25, pp. 3978-3984, 2007.

[10] M. Jhawer, S. Goel, A. J. Wilson, et al., "PIK3CA mutation/PTEN expression status predicts response of colon cancer cells to the epidermal growth factor receptor inhibitor cetuximab," Cancer Research, vol. 68, no. 6, pp. 1953-1961, 2008.

[11] A. Sartore-Bianchi, M. Martini, F. Molinari, et al., "PIK3CA mutations in colorectal cancer are associated with clinical resistance to EGFR-targeted monoclonal antibodies," Cancer Research, vol. 69, no. 5, pp. 1851-1857, 2009.

[12] G. Sarkar, J. Cassady, C. D. K. Bottema, and S. S. Sommer, "Characterization of polymerase chain reaction amplification of specific alleles," Analytical Biochemistry, vol. 186, no. 1, pp. 64-68, 1990.

[13] C. R. Newton, A. Graham, L. E. Heptinstall, et al., "Analysis of any point mutation in DNA. The amplification refractory mutation system [ARMS]," Nucleic Acids Research, vol. 17, pp. 2503-2516, 1989. 


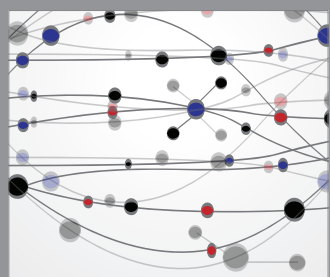

The Scientific World Journal
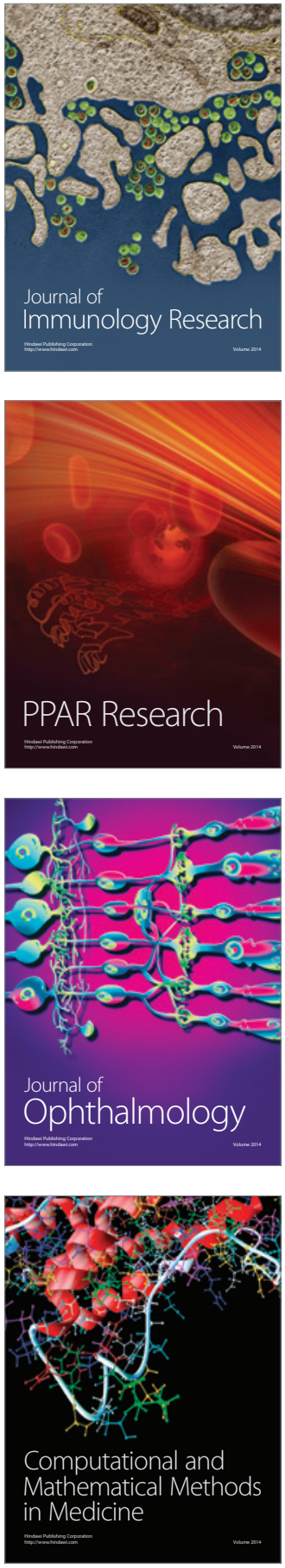

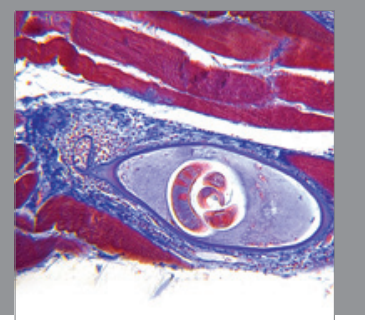

Gastroenterology

Research and Practice
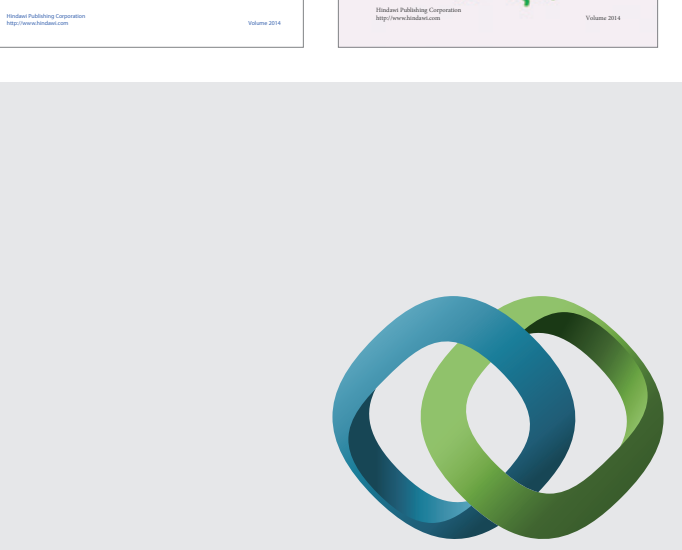

\section{Hindawi}

Submit your manuscripts at

http://www.hindawi.com
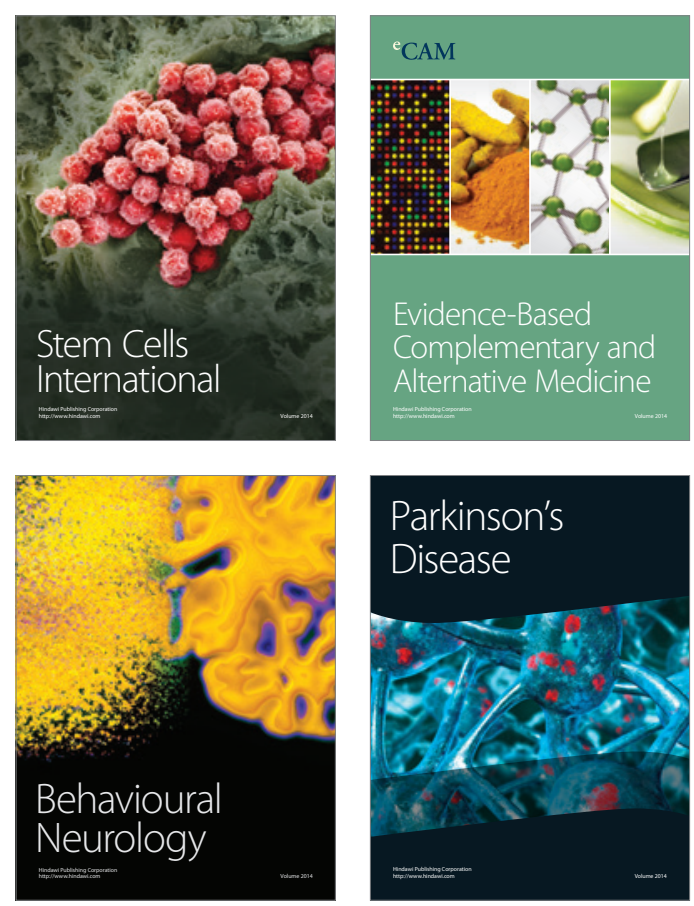

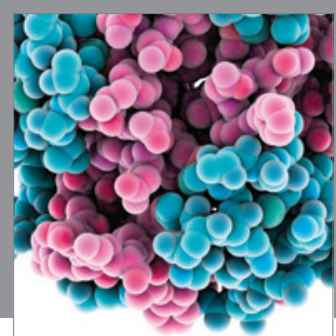

Journal of
Diabetes Research

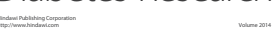

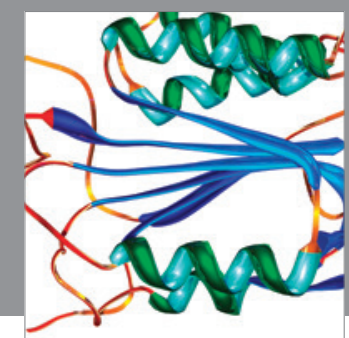

Disease Markers
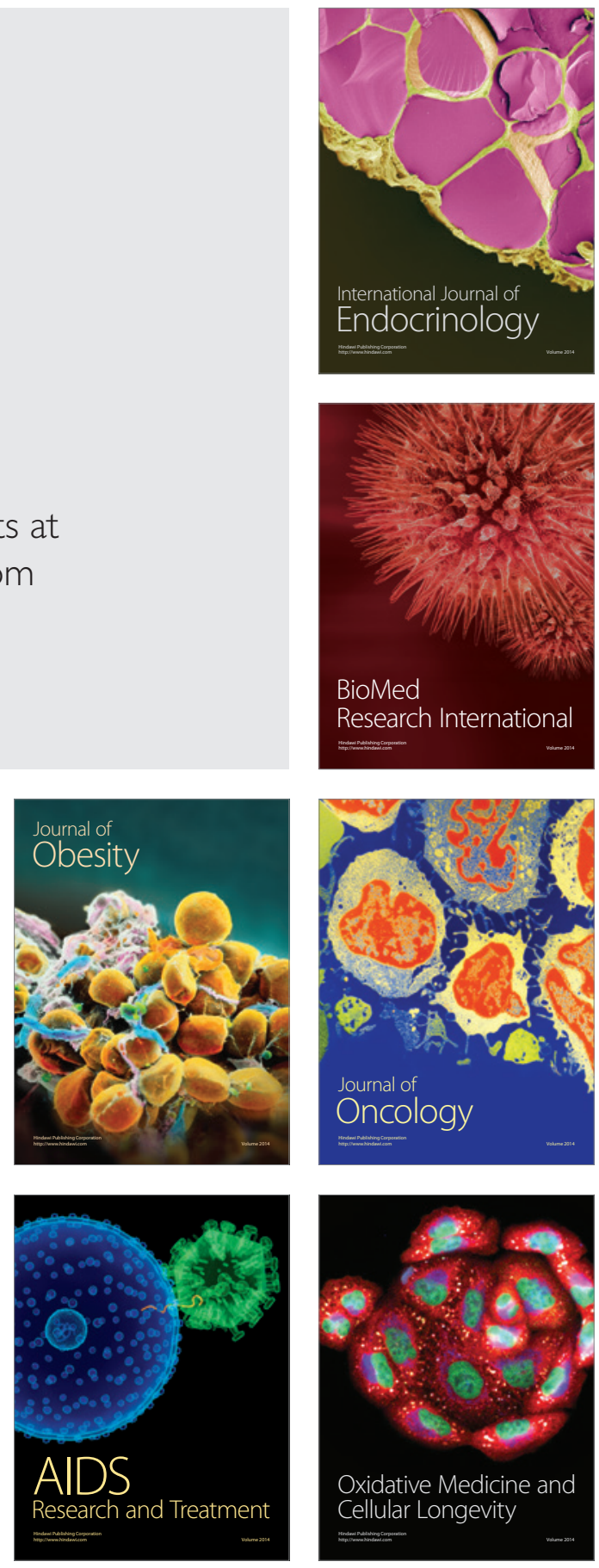